\title{
Study on Chinese Subjectivity and Chinese Grammar
}

\author{
Teaching
}

\author{
Kunhu $\mathrm{Wu}^{1}$ \\ ${ }^{1}$ Sichuan Agricultural University, College of Literature and Law, Yaan, Sicuan, \\ 625014
}

346591653@163.com

KEYWORDS: Chinese Subjectivity; Chinese Grammar Teaching

\begin{abstract}
In order to comply with the global boom in the ascendant Chinese learning, the state spent a lot of money in the world to establish a Confucius Institute and Confucius classroom, but also cultivate a batch after batch of outstanding foreign language teachers. So how do you teach Chinese language and dissemination of Chinese culture, which had to be attributed to the Foreign Language Teaching. Learning a language, to say the very fluently, you must master its grammar rules, the Foreign Chinese teaching, the Grammar Teaching is a top priority.
\end{abstract}

\section{Introduction}

Chinese say subjectivity, come to talk about what is subjective language. Subjectivity (subjectivity) refers to a characteristic of this language, that discourse is always more or less self-contained speaker performance components, the speaker at the same time to say some words, then show their position on this attitude and feelings, leaving the imprint of the self in discourse. Generally speaking subjectivity to language focused in three areas: the speaker's perspective; the speaker's emotions; the speaker's understanding. In recent years, linguists began subjective language given full attention, with the recent recovery of this linguistic humanism, in particular to functional linguistics, pragmatics, cognitive grammar the rise of so long been the dominant form of structural linguistics and linguistics advocated scientism be challenged. From these new schools are stressed, not only the objective language to express propositions and ideas, but also the expression of the body language of the speaker's point of view that is, feelings and attitudes.

\section{The Subjectivity in Chinese}

From the perspective of linguistic typology, language and some strong language belongs to subjectivity. For example, Japanese honorific system indicates that developed inevitably say when the Japanese use the precise form to express the speaker and the content of said listener's attitude or feelings. Many East Asian languages (including Chinese) with a kind of passive sentences more unhappy the 
Meaning, which is a manifestation of subjectivity. We mentioned above: the Chinese, how a Qing Wen sister did not put this kind of grammatical meaning of the words to express is a subjective disposition, Wang Mian-year-old father died of such sentences on the expression of the $\mathrm{Vi}+\mathrm{NP}$ the gains and losses is a subjective grammar sense, he is a child born last year in the form of so-called righteousness wrong with the sentence is grammatical expression meaning a subjective identity. This shows that, compared with English and other languages Chinese is more subjective language. We can even according to a language, there is no such representation, or according to their frequency of use, to determine the extent of the language level of subjectivity.

Speak a kind of sentence usage, talk about a three five grammatical rules, the student is likely to miss the forest for the trees, or in the elephant, the lack of overall sense of emotional grasp of the sentence. See Mr. Dong Qiao said in his prose: people are made of meat. . The text is made of meat. Modern civilized world gradually faded text function of this layer is always trying to text solidified into steel, into the plastic, inserts into the software and hardware being cold. He also said that the rules of grammar can be interpreted rational procedure, it may not explain got emotional spectrum. Cherry red, green banana is a rational statement; red cherry, green banana is sensual creation. In Chinese grammar teaching students to master Chinese characteristics syntax, an important job is to try to make students understand the important Chinese sentence contains the speaker's emotional spectrum. Some people may ask, in the past we used a grammatical rule Detailed analysis of the fine, is not doing're wrong? Or borrow Mr. Dong Qiao essays to answer. Water Margin in great length how to write and Pan Ximen hooked into adultery kill Wu Dalang, Wu Pan how to find ways to come back to conceal the truth, but just let Lion opera Pan exposing the inside to wear mourning clothes of red, immediately clear and simple indication inside there must be adultery. Mr. Dong Qiao commented that a detailed account of the ins and outs of the story, it is academic, with Hong Chang mourning under the key story points out, it is learned. I think our Chinese teachers certainly do not want to be in the academic and not a learned man.

\section{Chinese Grammar Teaching}

In addition to the choice of grammar teaching, Grammar Teaching specifically what to teach it? Overall it is teaching grammar points, including: a Chinese sentence; Second, the Chinese and foreign comparison; Third, syntax errors.

Study of sentence types, and is the focuses of a lot of people are concerned about. In foreign language teaching profession very seriously sentence of research and teaching, particularly in the preparation of Chinese textbooks, because the syntax included in the sentence, the sentence is how to teach very important. Foreign Language Teaching sentence is unlike the teaching of the tribe. Not only to correct analysis and interpretation of various structural characteristics sentence itself, but also pointed out to the students in what circumstances can this sentence. Among them, the Beijing Language Institute sentence Research Group (1989-1991) "Modern Chinese BASIC" is a large-scale, systematic research sentence, has very important reference 
value. In this paper, we collected more typical foreign language literature have 176 sentences, mainly in refining research on sentence, such as:

More research on the interrogative question of right and wrong, positive and negative aspects of questions and interrogative pronouns. A typical 12 on Interrogations article studies, in which there 4 interrogative pronouns, another typical snow Ding Huan wrote a series of articles on interrogative acquisition discussed Acquisition Interrogations. "Acquisition of Korean learners of Chinese Interrogations system", "to question students pronouns" who "question the use of non-use of research", "Foreign Language Teaching in Primary Stage interrogative how to effectively complete," "Foreign Language Teaching in doubt pronoun "how" questions about the use of non-teaching strategies" ," Chinese "what" non-interrogative usage of foreign teaching and research" ," Han Korean question words "a Comparative Study" based on the interrogative Interlanguage error analysis "," interrogative questions focus as a Foreign Language classroom questioning. "

This paper collected negative 12 sentence and those are mainly on the negative words, negative, negative aspects of the scope of the study. Jianqin studied the "no" and "no" in the acquisition process that is orderly, diffusion is a slow process, showing a continuous state of change at all stages. "Comparative Study of Korean negative sentences in Chinese" Han Chinese Negative Words and performance were compared. "Thai students learning Chinese Negative Sentence Errors research" conducted a study and comparison of negative sentences in Chinese and Thai, and Thai students investigate the causes negative sentence errors occur, also pointed out the corresponding countermeasures. "Grammar Teaching in Teaching negative word" that "no" "No" "Do not," "off" "No" "The difference between the characteristics of the non-syntax" "not" "No" and other negative words and their relationships, but also according to the characteristics of each negative word syntax, to take appropriate teaching methods in the Foreign Chinese Teaching. "Based on Teaching Chinese as a negative adverb" no "and" no "comparative analysis" comparative analysis "no" and the similarities and differences "No," the semantic function, and the students in the study cited "no" and "no" process typical errors that appear, and made corresponding teaching suggestions. Negative on Acquisition of sentences, both in-depth description of the process performance and verbal description, but also to analyze related errors occur, but remains to be further studied.

Comparative studies scholars sentence is more concerned, typically have 17 articles, there are studies in Chinese comparative sentences, but also Chinese and foreign comparative study of the two comparative sentence. For example: "Han Chinese Comparative Sentences contrast" by contrast, said Han Chinese things, character, the degree of similarities and differences of the sentence, found that Korean and Chinese languages compare different sentence structure, and further summarized in two languages compare differences in sentence structure corresponding laws. "Chinese to Vietnamese Students comparative sentences Error Analysis and Acquisition Order study" Comparative analysis of the Vietnamese students' Chinese Sentence Errors have more marked aspects of bias, bias comparative material respect, the conclusions term aspects of bias, but also in Vietnam Acquisition order 
comparative sentences class students were investigated, noting the impact of the target language, migration mother tongue, teaching materials, and learning strategies are all factors comparative sentences Acquisition. "Relatively junior level foreign students study Chinese Sentences" Through experiments and statistical analysis of the corpus of foreign students at the primary level geometric sentence, worse than the sentence, the sentence-level comparison of the Acquisition, and noted that the comparison of the impact of the Acquisition relevant factors. "Foreign students are more Sentences Errors Cause and Countermeasures" Comparative analysis of different foreign students the Acquisition Errors cultural backgrounds, different habits and language itself three reasons, and noted that appropriate measures: 1) serious Implication of Chinese language learning; 2) sentence drills; 3) the use of the mother tongue positive transfer; 4) pay attention to the word order. "Students Corpus of Chinese Comparative Sentences" by Beijing Language and Culture University inter-language corpus corpus, analyzes the right corpus of Chinese learners and used to explain the relatively common structure, with additional bias corpus analyzed the characteristics of Chinese comparative sentences .

\section{The Similarities and Differences In Syntax}

In the process of learning Chinese, due to different Chinese and native speakers of the target language learners, will inevitably make some grammatical problems, there is the case of most grammatical errors, Liu Xun in "Foreign Language Education Introduction" in the analysis of the emergence of Errors in five aspects, namely mother tongue negative transfer target language negative transfer of knowledge, learning and communication strategies and policies, study habits. Chinese learners of Chinese middle-class stage, due to their limited knowledge of Chinese grammar and pragmatic rules is not enough in-depth understanding, coupled with their ability to abstract and ability to imitate are relatively strong, they often use their own I learned knowledge to infer new knowledge. In addition to an aspect of teaching also appears Errors, currently used by Chinese learners of English textbooks are used most notes, which would have a lot of inconvenience. First, Chinese and English should not be accurate in terms of vocabulary and syntax, with English annotation is bound to cause deviation student understanding, such as "beautiful" and "beautiful" English comments have "beautiful" Such comments, bound to students doubt, confusion of its usage, even misuse; Secondly, many of the native language is not English learners in the learning process, they need to be translated into English should be understood in their native language, after two translators understood more likely to cause Errors and understanding of the issue of unclear use.

\section{Conclusion}

Chinese language teaching now achievements are basically engaged in domestic or foreign teachers teaching Chinese Foreign experience and related problems encountered by their actual teaching gained through research, provides us with a lot of lessons for later in the teaching of Chinese Foreign less take a lot of detours, it is 
worth as reference, since the grammar Teaching long way to go, need another batch of foreign language teachers to continue to pave the way, as first-line teachers, the most direct the contacts with foreign learners, this is the most important resources, and we should make full use of this advantage, the students' questions, and to explore on their own teaching have carried out some research, teaching for themselves and future generations provide some help.

\section{REFERENCE:}

[1] Chao, Yuan Ren.A Grammar of Spoken Chinese [M].Berkeley and Los Angeles: University of Califor-nia Press,1968.

[2] Finegan, Edward. Subjectivity and Subjectivisation: an introduction [M].In Stein, Dieter \& S.Wright,1995.

[3] Kuno, S. Functional Syntax: Anaphora, discourse and empathy [M].Chicago and London: Universi-ty of Chicago Press,1987.

[4] Lakoff, George. Women, Fire, and Dangerous Things [M].Chicago: University of Chicago Press,1987.

[5] Lyons, J.Semantics [M].Cambridge: Cambridge University Press,1977. 